\title{
Effect of the fluid-structure interaction on solid rocket motors instabilities
}

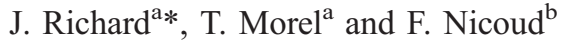 \\ ${ }^{a}$ Cerfacs, 42 Avenue Gaspard Coriolis, 31057 Toulouse, France; ${ }^{b}$ CNRS UMR5149, Université \\ Montpellier 2,2 Place Eugène Bataillon, 34095 Montpellier, France
}

Large solid propellant rocket motors may be subjected to aero-acoustic instabilities arising from a coupling between the burnt gas flow and the acoustic eigenmodes of the combustion chamber. Given the size and cost of any single firing test or launch, it is of first importance to predict and avoid these instabilities at the design level. The main purpose of this paper is to build a numerical tool in order to evaluate how the coupling of the fluid flow and the whole structure of the motor influences the amplitude of the aeroacoustic oscillations living inside of the rocket. A particular attention was paid to the coupling algorithm between the fluid and the solid solvers in order to ensure the best energy conservation through the interface. A computation of a subscaled version of the Ariane 5 solid propellant engine is presented as illustration.

Les moteurs à propergol solide sont parfois le siège d'instabilités aéro-acoustiques résultant d'un couplage entre l'hydrodynamique des gaz brûlés et les modes acoustiques de la chambre de combustion. Au vu du coût d'un essai, il est important de pouvoir prédire l'apparition de ces instabilités au moment de la conception. L' objectif de cette étude est la mise au point d'une chaîne de couplage permettant d'évaluer l'impact des interactions fluide-structure sur l'amplitude des oscillations aéroacoustique présentes au sein du propulseur. Une attention particulière a été portée à l'algorithme de couplage entre les solveurs fluide et solide afin d'assurer une bonne conservation de l'énergie à l'interface fluide-structure. Une simulation d'une échelle réduite du moteur d'Ariane 5 est présentée comme illustration.

Keywords: large eddy simulation; Arbitrary Lagrangian Eulerian method; fluid-structure interaction

Mots-clés: simulation aux grandes echelles; maillage mobile; interactions fuilde-structure

\section{Introduction}

The Ariane 5 solid rocket motor (SRM), named P230, is one of the most impressive solid rocket motors ever constructed in Europe. It is about $37 \mathrm{~m}$ high for a radius of $1.5 \mathrm{~m}$, with an initial mass of about 280 tonnes. The two SRMs represent $90 \%$ of the thrust at take-off, burning out during $130 \mathrm{~s}$ and consuming approximately 2 tonnes of propellant each second. They are finally ejected when the rocket has reached an altitude of approximately $70 \mathrm{~km}$ and a speed of $2000 \mathrm{~m} / \mathrm{s}$. Their corresponding thrust is unfortunately not stationary during the firing, and some oscillations may occur. This might jeopardise the integrity of the payload due to vibrations. The phenomenon has been investigated extensively over the last decades (Culick,

*Corresponding author. Email: richard@cerfacs.fr 


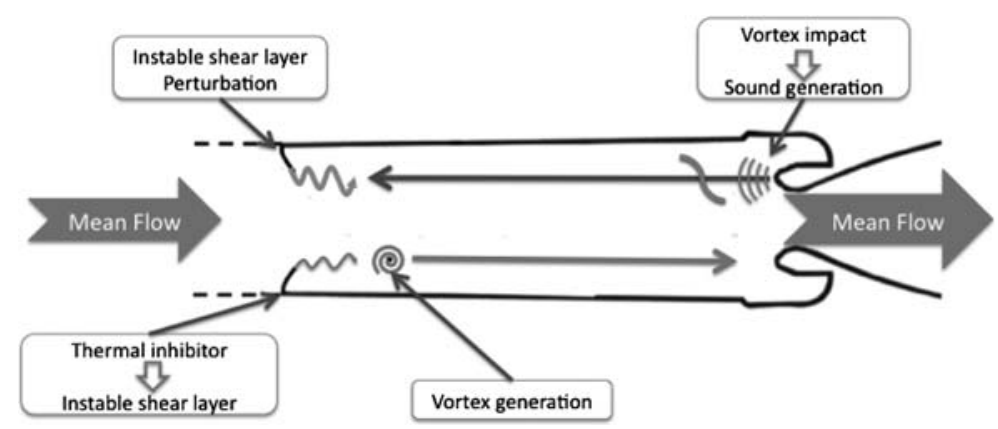

Figure 1. Instabilities mechanism.

1966; Fabignon et al., 2003; Flandro, 1986; Kourta, 1996a, 1996b; Lupoglazoff \& Vuillot, 1996; Vuillot, 1995). This mechanism is a coupling between the acoustic mode and the hydrodynamic perturbation, as represented in Figure 1. An unstable shear layer in the mean flow produces vortices which are convected until they impact the head of the nozzle. The acoustic wave generated by this impact can move back upstream since the flow is subsonic. It perturbs the unstable shear layer, intensifying the generation of vortices. Such an aero-acoustic mechanism can lead to high-amplitude fluctuations when the underlying frequency is close to the frequency of an acoustic mode of the whole geometry. Note that another instability mechanism relying on the intrinsic hydrodynamic instability of the boundary layer which develops over the propellant burning surface can also lead to significant pressure oscillations (Lupoglazoff \& Vuillot, 1996). As far as the P230 SRM is concerned, the experiment shows that mainly the first and second acoustic modes are excited during three outbursts, the first being the stronger one.

\section{Presentation of the numerical software}

Classically, the fluid-structure interaction (FSI) problem consists of solving simultaneously both the fluid and the structural equations where some variables of one act as a boundary condition for the other. Formally, the coupled fluid-structure problem may be written as:

$$
\begin{gathered}
\frac{\mathrm{d}}{\mathrm{d} t}(A W)+F^{c}(W, x, \dot{x})=R(W, x) \\
M \frac{\mathrm{d}^{2}}{\mathrm{~d} t}(U)+D \frac{\mathrm{d}}{\mathrm{d} t}(U)+K U=f^{e x t}(W(x, t), x)
\end{gathered}
$$

In this semi-discrete formulation already presented by Lesoinne (Lesoinne \& Farhat, 1993), dots stand for time derivatives, $x$ is the displacement or position vector of the fluid grid points, $W$ is the fluid state vector, $A$ results from the discretisation of the flow motions equations, $F^{c}=F-\dot{x} W$ is the vector of Arbitrary Lagrangian Eulerian (ALE) convective fluxes, $F$ is the vector of convective fluxes, $R$ is the vector of diffusive fluxes, $U$ is the structural displacement vector and finally $M, D$ and $K$ are, respectively, the mass, damping and stiffness matrices of the structural system. In order to solve the problem of FSIs, we have chosen to use a Conventional Serial Staggered (CSS) method with subcycling (Farhat \& Lesoinne, 1996; Piperno \& Farhat, 2001; Piperno, Farhat, \& Larrouturou, 1995). Softwares we used are shortly presented in this section, then the CSS method is reminded and the mesh movement technique is explained. 


\subsection{Fluid solver}

Two different modelling tools are used for this work. First of all, aero-acoustics calculations are performed with the large eddy simulation (LES) solver AVBP (Schoenfeld \& Rudgyardt, 1999) developed at CERFACS and IFP Energies nouvelles and widely validated for aero-acoustic applications (Schmitt, Poinsot, Schuermans, \& Geigle, 2007). AVBP is designed to solve the Navier-Stokes equations for three-dimensional compressible flows over unstructured meshes. The numerical results presented in this paper correspond to a Lax-Wendroff scheme which is 2nd order in both space and time. Note however that the methodology developed is compatible with any of the more accurate schemes available in AVBP (3rd order, 4th order). The Lax-Wendroff scheme was used in this preliminary study to reduce the CPU time.

\subsection{Structure solver}

The structural deformations are computed with the structural analysis software MARC, using a finite element method (FEM) and developed by MSC-Software. It is well suited for the treatment of non-linear materials, commonly encountered in solid propulsion. It allows static, dynamic and modal computations. The numerical scheme used in our computations is the trapezoidal Newmark method (see Equation (3)) (Hughes, 1987), which has already been advocated for coupling applications (Giordano et al., 2005; Piperno \& Farhat, 2001). It consists of writing the structure's equilibrium at the time $t^{n+1}$, knowing its state at $t^{n}$ and the external force $f^{n+1}$ at $t^{n+1}$ :

$$
\left(\frac{4}{\Delta t_{\mathrm{s}}^{2}} M+\frac{2}{\Delta t_{\mathrm{s}}} D+K\right) U^{n+1}=f^{n+1}+\left(\frac{4}{\Delta t_{\mathrm{s}}^{2}} M+\frac{2}{\Delta t_{\mathrm{s}}} D\right) U^{n}+\left(\frac{4}{\Delta t_{\mathrm{s}}} M+D\right) \dot{U}^{n}+M \ddot{U}^{n}
$$

\subsection{Coupling algorithm}

In most cases, the time step $\Delta t_{\mathrm{f}}$ for the fluid is much smaller than the structural one $\Delta t_{\mathrm{s}}$. Then, we consider that the coupling time step $\Delta t_{\mathrm{c}}$ is equal to $\Delta t_{\mathrm{s}}$. In the CSS method (Farhat \& Lesoinne, 1996; Piperno et al., 1995; Piperno \& Farhat, 2001), each coupling iteration which allows to advance the solution from $t^{n}$ to $t^{n+1}=t^{n}+\Delta t_{\mathrm{s}}$ consists of four steps:

(1) Predict the structural displacement $u^{n+1 p}$ at time $t^{n+1}$. A common predictor is:

$$
u^{n+1 p}=u^{n}+\alpha_{0} \Delta t_{\mathrm{s}} \dot{u}^{n}+\alpha_{1} \Delta t_{\mathrm{s}}\left(\dot{u}^{n}-\dot{u}^{n-1}\right)
$$

It is worth noticing that the choice of $\alpha_{0}=1$ and $\alpha_{1}=0$ corresponds to a first-order predictor, while $\alpha_{0}=1$ and $\alpha_{1}=1 / 2$ defines a second-order one.

(2) Advance the fluid system to $t^{n+1}=t^{n}+\Delta t_{\mathrm{S}}$ while updating the position of the fluid grid in order to match the position $u^{n+1 p}$ at the end of the coupling steps. Because $\Delta t_{f}<\Delta t_{\mathrm{s}}$ this step is achieved by subcycling the fluid solver (typically in our computations, one structural iteration is performed every 100 fluid iterations).

(3) Transfer the fluid pressure $P_{\mathrm{S}}^{n+1}$ to the structure. Note that $P_{\mathrm{S}}^{n+1}$ is not necessarily the fluid pressure at the interface at time $t^{n+1}$. Many choices for $P_{\mathrm{S}}^{n+1}$ can be found in the literature. The instantaneous pressure field of the fluid at the interface or its integral 
over the subcycled time are common examples. The impact of this choice is discussed in a later section.

(4) Finally integrate the structure to $t^{n+1}$ with the external force $f^{n+1}$ of Equation (2) being deduced from $P_{\mathrm{S}}^{n+1}$.

The OpenPALM coupler co-developed by CERFACS and ONERA is used to synchronise the fluid and structure solvers (Buis, Piacentini, \& Déclat, 2006). It also provides data transmission between the different tools at the fluid-structure interface without large intrusion in the different solvers. As a first step, the interpolation between the non-conformal meshes is achieved with a first-order method.

\subsection{Mesh deformation}

A classical issue in fluid computation with deformable domain is the mesh deformation when the displacement of the boundary is unknown by advance, which is typically the case in fluid-structure computations. Many methods designed to transmit the movement of the boundary to the internal nodes can be found in the literature. The most popular are the linear spring analogy (Batina, 1990), the torsional spring method (Farhat, Degand, Koobus, \& Lesoinne, 1998) or the pseudo-solid approach (Nielsen \& Anderson, 2002). The method we choose to implement in AVBP consists of requiring that the displacement $\mathrm{d} x_{i}$ of every node $i$ is the weighted average of the displacement of its surrounding vertices $j$.

$$
\mathrm{d} x_{i}=\frac{1}{\sum_{j} \alpha_{j}} \sum_{j} \alpha_{j} \mathrm{~d} x_{j}
$$

Classical weight $\alpha_{j}$ is the inverse of the distance between the nodes $i$ and $j$. This set of equations can be formulated as a linear problem $S \mathrm{~d} x=b$, where $b$ contains the contribution of the boundary nodes, which have an imposed displacement. This is solved with a standard conjugate gradient approach. The conjugate gradient method is never fully converged, but instead is run until the residuals have decreased to the limit defined by the user. The mesh deformation is taking into account in AVBP with the classical ALE equations. In order to ensure the Geometric Conservation Law (GCL) (Lesoinne \& Farhat, 1996), the velocity of the fluid/structure interface is kept constant during the subcycling and is defined as $\frac{u^{n+1 p}-u^{n p}}{\Delta t_{\mathrm{s}}}$.

\section{1D acoustic configuration for code verification}

Aero-acoustic instabilities are known to be extremely sensitive to energy loss and gain. When considering the impact of FSI on such instabilities, it is thus mandatory to make sure that the coupling algorithm does not add/remove energy to the system. To this respect, a specific test case was designed in order to demonstrate the capability of the numerical chain to couple the fluid and solid domains in a virtually conservative way.

\subsection{Set-up description}

The set-up is composed of an adiabatic chamber filled with gas, closed at the left-hand side by a fixed wall and at the right-hand side by a deformable block of rubber which is fixed on its right side, see Figure 2. The fluid chamber has a section $S_{0}$ and length $l_{0}$ when the block of rubber is at rest at $x=l_{0}$. The rubber of density $\rho_{\mathrm{S}}$ has a Young modulus $E$ and a Poisson ratio 


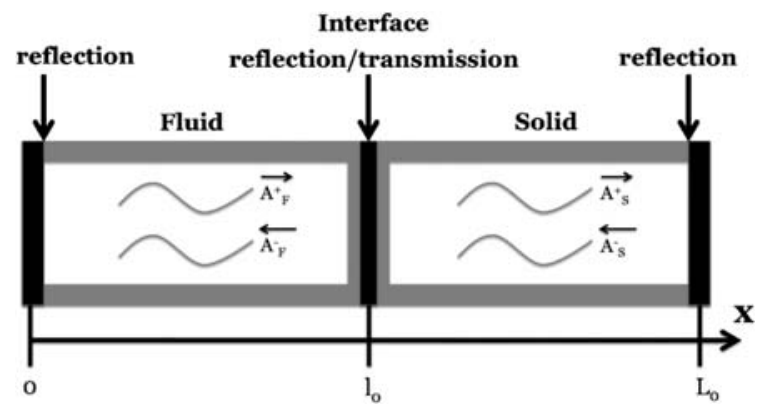

Figure 2. Acoustic coupling system.

$v$, and is fixed at $x=L_{0}$. Displacement (or position, depending on the context), velocity and acceleration in the solid at time $t$ are, respectively, $U(t), \dot{U}(t)$ and $\ddot{U}(t)$. Starting from rest, the fluid part is then disturbed with a pressure Dirac impulsion. After some iterations, coupled eigenfrequencies appear while looking at time evolution of the pressure signal in the chamber.

The following assumptions are first made for the system:

- The gas is perfect.

- Dissipation is neglected in both fluid and solid.

- The deformations of the solid are small, which allow to approximate the interface location by $l_{0}$.

\subsection{Acoustic modelling}

The following expressions can be obtained for the speed of propagation in the fluid $\left(C_{\mathrm{F}}\right)$ and solid $\left(C_{\mathrm{S}}\right)$, respectively:

$$
C_{\mathrm{F}}=\sqrt{\gamma R T} \quad C_{\mathrm{S}}=\sqrt{\frac{(1-v) E}{\rho_{\mathrm{S}}(1+v)(1-2 v)}}
$$

Then, $P$ being the oscillating pressure and $\dot{U}$ being the oscillating velocity, the following wave decomposition holds for both fluid and solid:

$$
\begin{gathered}
P_{\mathrm{F}, \mathrm{S}}=A_{\mathrm{F}, \mathrm{S}}^{+} e^{j k_{\mathrm{F}, \mathrm{S}} x}+A_{\mathrm{F}, \mathrm{S}}^{-} e^{-j k_{\mathrm{F}, \mathrm{S}} x} \\
\dot{U}_{\mathrm{F}, \mathrm{S}}=\frac{1}{\rho_{\mathrm{F}, \mathrm{S}} C_{\mathrm{F}, \mathrm{S}}}\left(A_{\mathrm{F}, \mathrm{S}}^{+} e^{j k_{\mathrm{F}, \mathrm{S}} x}-A_{\mathrm{F}, \mathrm{S}}^{-} e^{-j k_{\mathrm{F}, S} x}\right)
\end{gathered}
$$

where $K_{\mathrm{F}, \mathrm{S}}=\omega / C$ is the wave number. Using (6) and (7) along with the appropriate boundary and jump conditions:

- $\dot{U}_{\mathrm{F}}(0)=\dot{U}_{\mathrm{S}}(0)=0$ (impermeable wall condition for the fluid and embedding for the solid).

- $\dot{U}_{\mathrm{F}}\left(l_{0}\right)=\dot{U}_{\mathrm{S}}\left(l_{0}\right)$ (velocity continuity at the fluid-structure interface located at $l_{0}$ ). 
- $P_{\mathrm{F}}\left(l_{0}\right)=P_{\mathrm{S}}\left(l_{0}\right)$ (stress continuity at the fluid-structure interface located at $l_{0}$ ).

It follows that any eigen angular frequency $\omega_{c}$ is solution of the following dispersion relation (8):

$$
\frac{\tan \left(\frac{\omega_{\mathrm{c}}}{C_{\mathrm{S}}}\left(l_{0}-L_{0}\right)\right)}{\tan \left(\frac{\omega_{c}}{C_{\mathrm{F}}}\left(l_{0}\right)\right)}=\frac{\rho_{\mathrm{S}} C_{\mathrm{S}}}{\rho_{\mathrm{F}} C_{\mathrm{F}}}
$$

This relation allows to find the coupled eigenmodes of the system which, depending from the sub-systems properties, may differ from the eigenfrequency of the fluid and solid taken alone.

\subsection{Energy consideration}

Following Piperno and Fahrat (Piperno \& Farhat, 2001), it is possible to calculate the numerical amount of energy imbalance created at the fluid-structure interface $\Delta E$ in one oscillation. According to their results $\Delta E \sim \pi\left(\delta E_{\mathrm{F}}+\delta E_{\mathrm{S}}\right)$, where $\delta E_{\mathrm{F}}$ can be interpreted as an energy transferred from the fluid to the structure as viewed from the fluid and $\delta E_{\mathrm{S}}$ is the same energy but as viewed from the solid. In our particular case with subcycling, we have (after some algebra):

$$
\delta E_{\mathrm{F}}=k_{\mathrm{e}}\left[\left(\alpha_{0}-1\right) \zeta+\left(\frac{1}{4}-\frac{7 \alpha_{0}}{12}+\frac{3 \alpha_{1}}{2}\right) \zeta^{3}\right]+O\left(\zeta^{4}\right)
$$

where $k_{\mathrm{e}}=\frac{-4 A_{\mathrm{G}}^{+2}}{\rho_{\mathrm{G}} C_{\mathrm{G}} \omega_{\mathrm{c}}} \sin \left(k_{\mathrm{G}} l_{0}\right) \cos \left(k_{\mathrm{G}} l_{0}\right), \zeta=\omega \Delta t_{\mathrm{c}}$ and $\alpha_{0,1}$ are the coefficients used by the predictor given in Equation (4). Besides, $\delta E_{\mathrm{S}}$ depends on the choice of the pressure transmitted to the structure $P_{\mathrm{S}}^{n+1}$ (see Section 2.3). Specifically one shows that:

- For the choice $P_{\mathrm{S}}^{n+1}=P^{n+1}$ (transmission of the updated pressure):

$$
\delta E_{\mathrm{S}}=O\left(\zeta^{4}\right)
$$

- For the choice $P_{\mathrm{S}}^{n+1}=\frac{1}{\Delta t_{\mathrm{c}}} \int_{t^{n}}^{t^{n+1}} P(t) \mathrm{d} t$ (transmission of the averaged pressure):

$$
\delta E_{\mathrm{S}}=k_{\mathrm{e}}\left[-\frac{\zeta}{2}+\frac{\zeta^{3}}{8}\right]+O\left(\zeta^{4}\right)
$$

Using a first-order predictor (which means $\alpha_{0}=1$ and $\alpha_{1}=0$ ), the choice of Equation (10) for $P_{\mathrm{S}}^{n+1}$ produces energy at third order, which is not an issue with regard to the secondorder accuracy of the fluid and structural solvers. On the other hand, if $k_{\mathrm{e}}<0$, the choice of Equation (11) for $P_{\mathrm{S}}^{n+1}$ will create energy at the first order. In this case, the coupling of the fluid and structure solvers induces an error as demonstrated in the following section.

\subsection{Numerical results}

Both fluid and structure are modelled in two dimensions for this test case. Since only longitudinal 1D solution are sought for, symmetric conditions have been used for the fixed bottom and top walls. The solid is modelled in MARC by a bloc of material of Young's Modulus $E=4.274 \times 10^{5} \mathrm{~Pa}$ and Poisson's coefficient $v=0$ such that its first longitudinal acoustic 
mode is of the same order than the first acoustic mode of the cavity (cf. Figure 3). It has a section $S_{\mathrm{M} 0}=S_{0}$ and an unstretched length $L_{\mathrm{M} 0}$. The calculations based on the first and second choices of $P_{\mathrm{S}}^{n+1}$ have been achieved using coupling time step 10 times bigger than the fluid one (i.e. $\Delta t_{\mathrm{c}}=\Delta t_{\mathrm{s}}=10 \Delta t_{\mathrm{f}}$ ). It is worth noticing in Figure 3 that the choice of Equation (10) for $P_{\mathrm{S}}^{n+1}$ presents a good capacity to retrieve the coupling eigenfrequency of the system and that the calculation appears to be stable. Looking at Figure 4 at the pressure signal filtered around $400 \mathrm{~Hz}$, one can notice that the coupled mode at $400 \mathrm{~Hz}$ appears to be energised with the choice of Equation (11). This result is coherent with the energy consideration of Section 3.3. Writing pressure and displacement at the fluid-structure interface for one of the coupled eigenmodes shows that the pressure and the structure are vibrating at the same angular frequency $\omega_{\mathrm{c}}$ but with a phase depending on $k_{\mathrm{G}}$ and $l_{0}$. It can be checked the scalar $k_{\mathrm{e}}$ (see Equation (9)) from the previous section is negative when $\sin \left(\frac{2 \omega_{\mathrm{c}} l_{0}}{C_{\mathrm{G}}}\right)>0$. It comes out from this analysis that the second choice of $P_{\mathrm{S}}^{n+1}$ feeds in energy some modes and damp the others, as illustrated in Figure $4\left(\sin \left(\frac{2 \omega_{\mathrm{c}} l_{0}}{C_{\mathrm{G}}}\right) \sim 1\right.$ for $\left.f_{\mathrm{c}}=400 \mathrm{~Hz}\right)$.

\section{Application to the subscaled P230 configuration with FSI}

\subsection{Computations global description}

The numerical chain described previously is now applied to a case representative of the industrial complexity which has already been a subject of interest for fluid-structure interaction
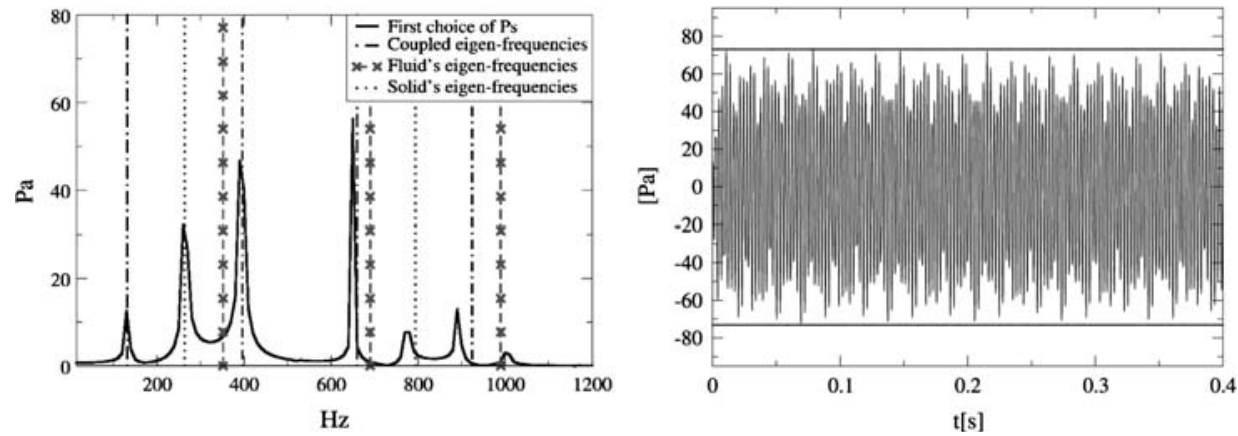

Figure 3. Fourier Transform (on the left-hand side) of the pressure signal at the middle of the chamber and its filtered version around $400 \mathrm{~Hz}$ (on the right-hand side) for the choice of Equation (10) for $P_{\mathrm{S}}^{n+1}$.
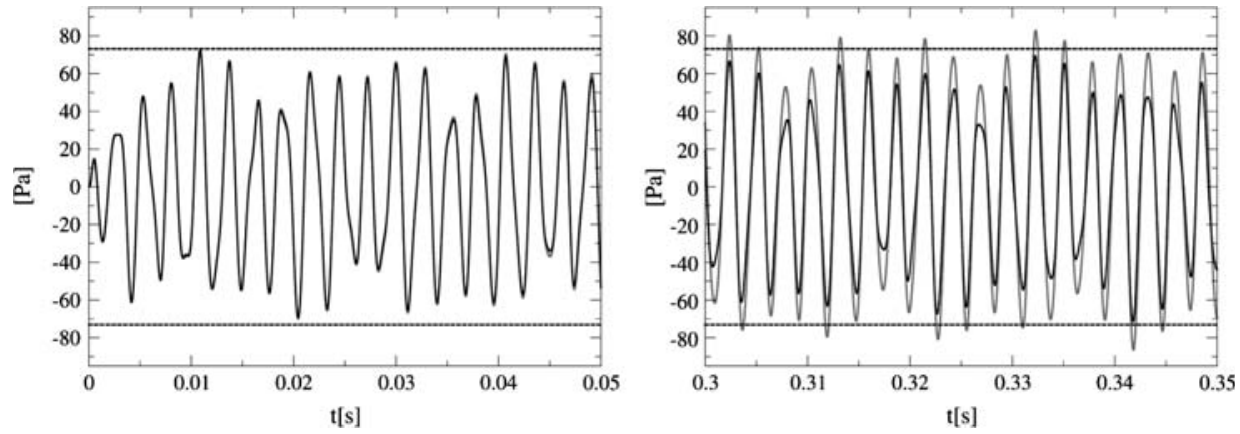

Figure 4. Pressure signal at the middle of the chamber filtered at $400 \mathrm{~Hz}$ for the choice of Equation (10) (black line) and Equation (11) (grey line) for $P_{\mathrm{S}}^{n+1}$. 
research (Dotson \& Sako, 2004; Wasistho, Fiedler, Namazifard, \& Mclay, 2006). The computational domain (fluid and structure) correspond to the P230 SRM at scale 1/15, thus considering a system in which size is comparable to the subscaled set-up used for past studies carried out by ONERA (Hijlkema, Prévost, \& Casalis, 2011). Note also that the 2D axisymmetric approximation is made to save CPU time and because it allows to illustrate the operability of the numerical chain. The geometry retained corresponds to $86 \mathrm{~s}$ combustion time of the full-scale P230; this corresponds to the second out of three outbursts. The chamber geometry was provided by Herakles. The material characteristics (such as the density or the Young modulus) of the structure were adjusted to reproduce the same crossover of eigenmodes between the fluid and solid domains as at full scale. Different materials, such as propellant, steel and thermal inhibitors, are considered in the computation, each with its own mechanical properties, as provided by Herakles. The structure is fixed at the front-end in this preliminary study. The global mechanical computational domain is displayed in Figure 5. Frontal thermal inhibitors were considered rigid in the presented fluid-structure computations. Still, the shape of the thermal inhibitor between the second and the third blocs of propellant was estimated in a pre-computation in order to account for its mean deflection due to the mean flow rate. This was done by performing a FSI computation with the same numerical chain as described above. Note that the dissipation of the inhibitor was artificially increased in this pre-computation in order to speed up the convergence to a steady position. Left-hand side of Figure 6 displays the initial shape and the final deflection of the frontal thermal inhibitor, while the displacement convergence of its end-point is displayed on the right-hand side. The acoustic mode of the combustion chamber has been determined thanks to the AVSP Helmholtz solver (Nicoud, Benoit, Sensiau, \& Poinsot, 2007). Results for the first and second longitudinal acoustic modes are displayed in Figures 7 and 8. Frequencies are, respectively, 308 and $611 \mathrm{~Hz}$.

\subsection{Numerical results}

Three calculations are presented here. The first one is done without FSI in order to produce a reference case. The second and the third calculations account for the structural response of

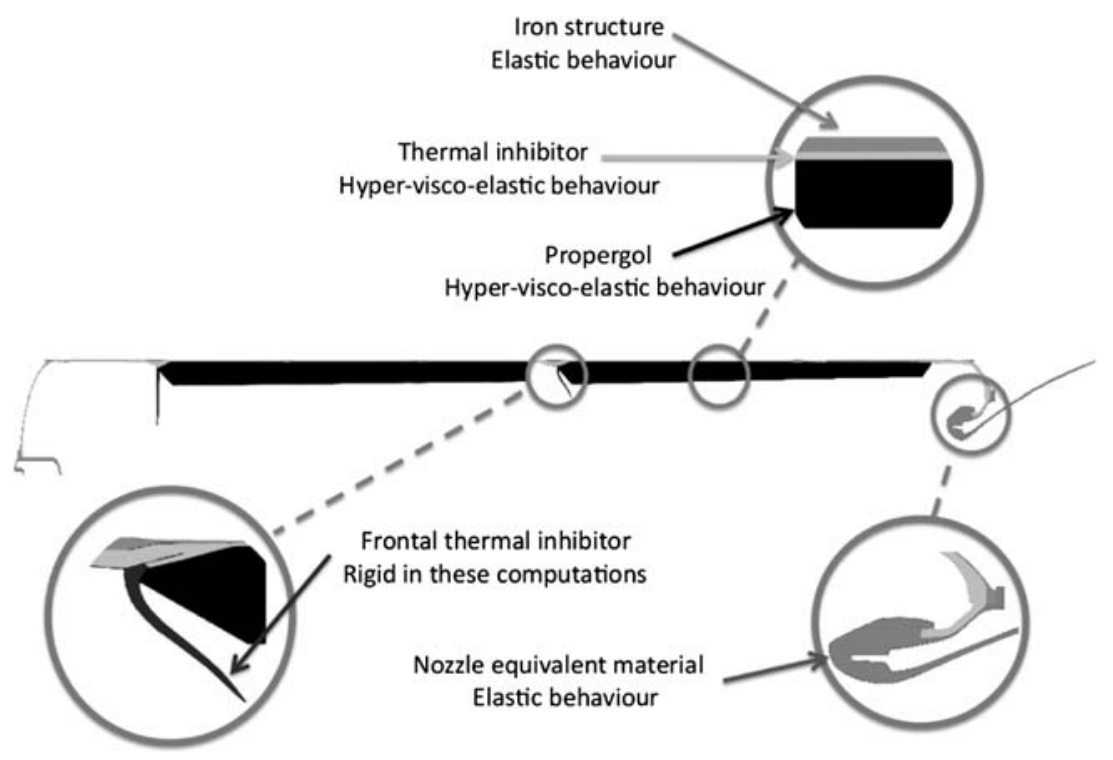

Figure 5. Unscaled figure of the P230 configuration. 

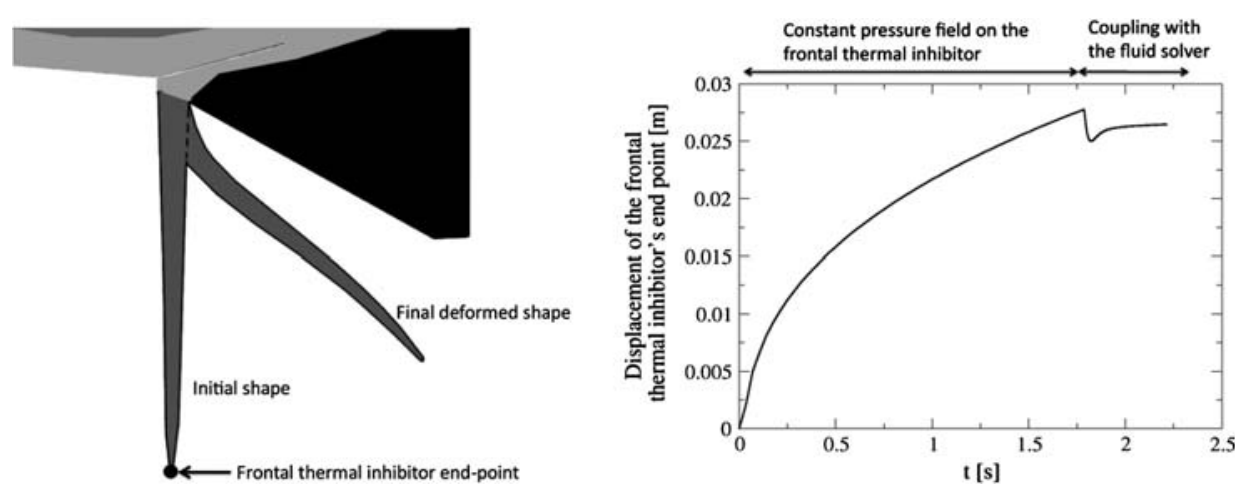

Figure 6. Initial and deformed shape of the thermal inhibitor between the second and the third blocs of propellant (left) and its end-point displacement during the simulation (right).

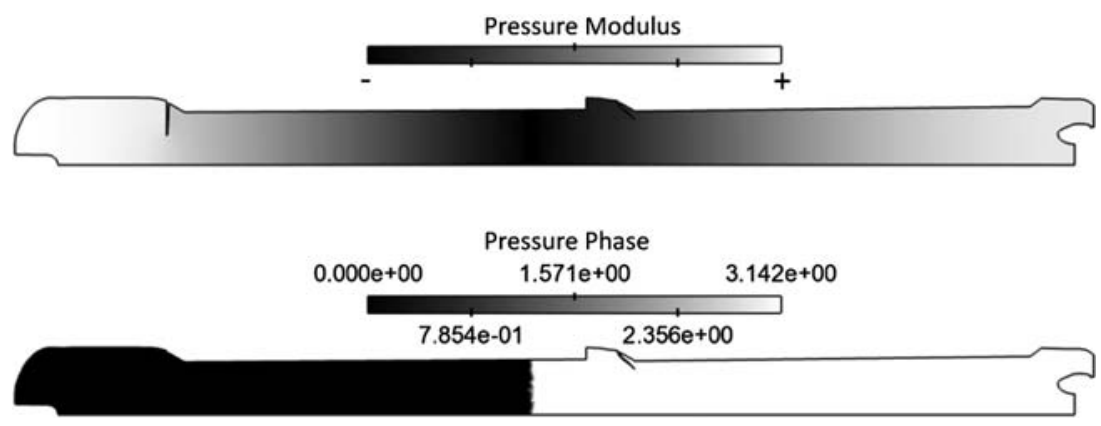

Figure 7. First longitudinal acoustic mode at $308 \mathrm{~Hz}$.
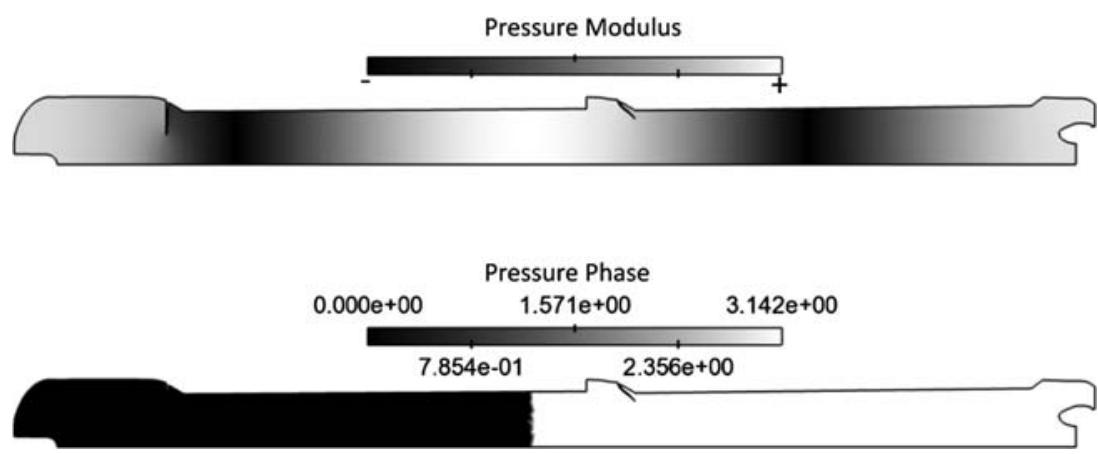

Figure 8. Second longitudinal acoustic mode at $611 \mathrm{~Hz}$.

the P230. When accounting for the interaction with the structure two situations can be considered. On the one hand, the first acoustic mode and the first longitudinal mode of the structure can be different (handled in the second calculation). On the other hand, they can be identical (handled in the third calculation). In both cases, the material dissipation was arbitrary fixed to be $D=10^{-5} K$ (recall $K$ is the stiffness matrix, see Equation (2)). A snapshot of the flow obtained by solving the full Navier-Stokes equations is displayed in Figure 9. It shows the presence of vortices generated near the propellant and at the extremity of the thermal inhibitor 


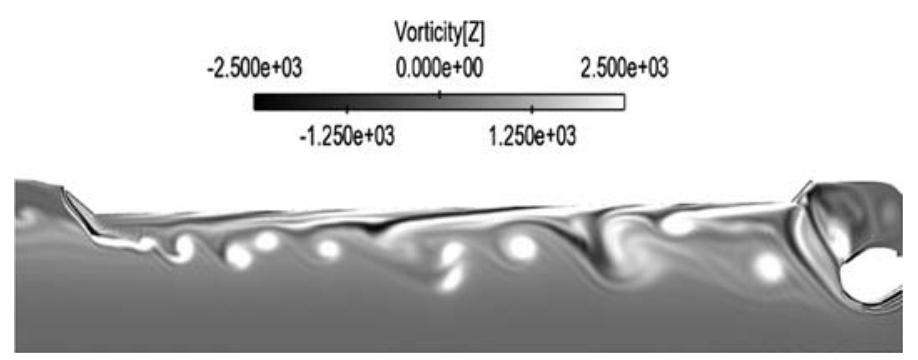

Figure 9. Unscaled figure of the vorticity generated near the propellant and at the thermal inhibitor.

during the calculation. The comparison of the aft-end vibration speed is presented in Figure 10. As suggested by the intuition, the vibrations are stronger when the structural mode happened to be at the same frequency as the acoustic mode.

The pressure at front-end is a classical measurement for aero-acoustic instabilities in SRMs. Comparisons between the case without fluid-structure interaction and the two cases taking into account the structural response are displayed in Figures 11-13. Pressure spectra at front-end (not shown) display coherent results between the LES and the acoustic solver, the first and second acoustic modes at 308 and $611 \mathrm{~Hz}$ being present in the LES results. Their

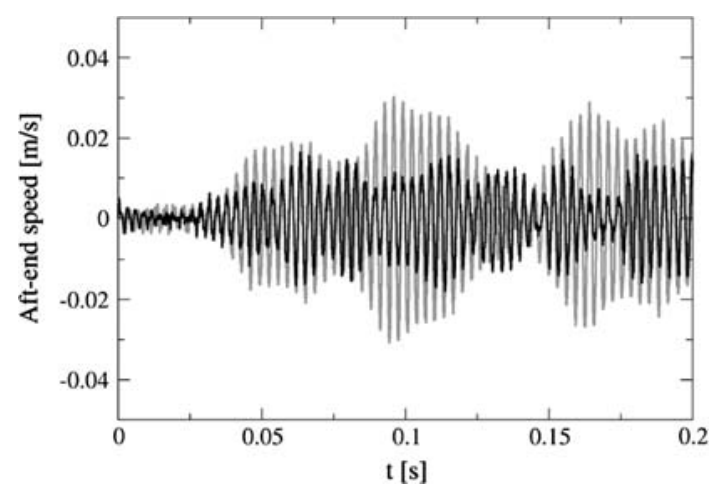

Figure 10. Velocity of the aft-end for the case with (grey line) and without (black line) crossing of the acoustic and structural modes.

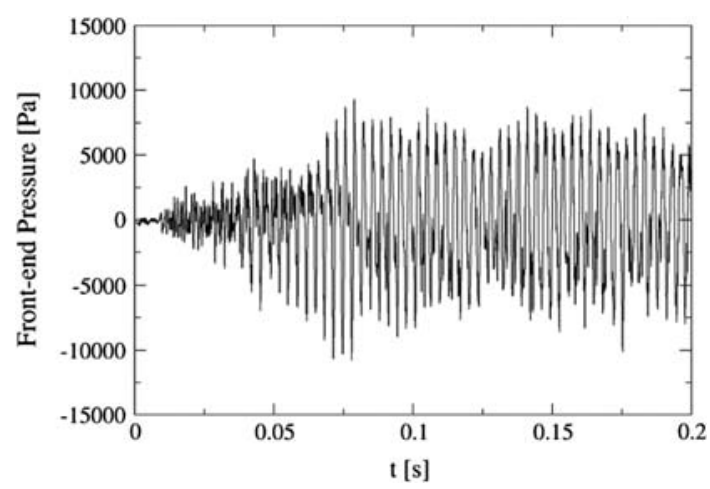

Figure 11. Pressure fluctuations at front-end for the case without FSI. 


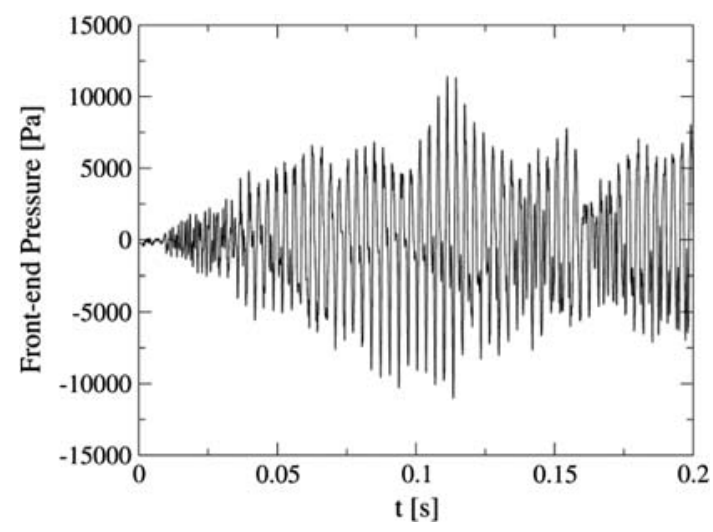

Figure 12. Pressure fluctuations at front-end for the case without crossing of the acoustic and structural modes.

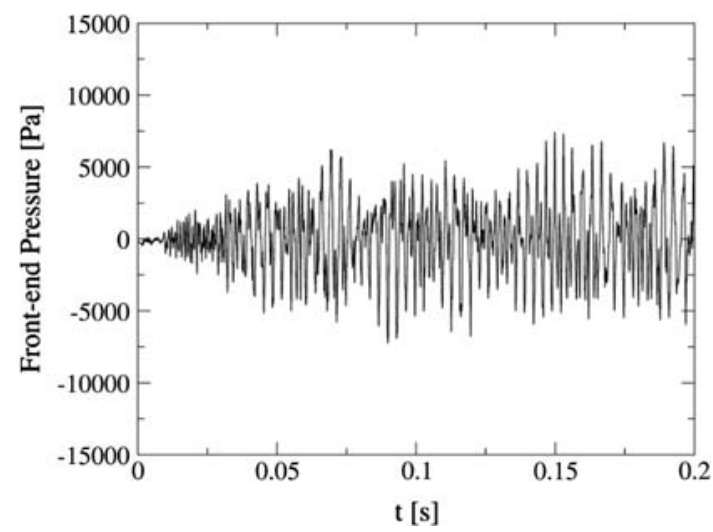

Figure 13. Pressure fluctuations at front-end for the case with modes crossing of the acoustic and structural modes.

corresponding amplitudes are displayed in Figure 14. Amplitudes are evaluated through the integration of a power spectral density of the signal over frequency ranges which are:

- $250-350 \mathrm{~Hz}$ for the first acoustic mode.

- $550-650 \mathrm{~Hz}$ for the second acoustic mode.

Surprisingly, results show that the acoustic mode is damped when the structural and acoustic modes happened to cross. Explanation is that the displacement amplitude being greater when the structural and acoustic modes are identical, the dissipation in the material plays a greater role due to larger deformation speed within the structure. The structure in this computation finally appears to act as a band-stop filter for the fluid, the rejected frequency being the first structural eigenmode. This interpretation is confirmed by a fourth simulation where the first acoustic mode and the first longitudinal mode of the structure are also identical but the dissipation of the materials is fixed to a lower value $\left(D=10^{-7} \mathrm{~K}\right.$ instead of $\left.10^{-5} \mathrm{~K}\right)$. The corresponding front-end pressure signal is displayed in Figure 15. Although, the vibrations of the structure are stronger for the calculation with less-dissipative materials 


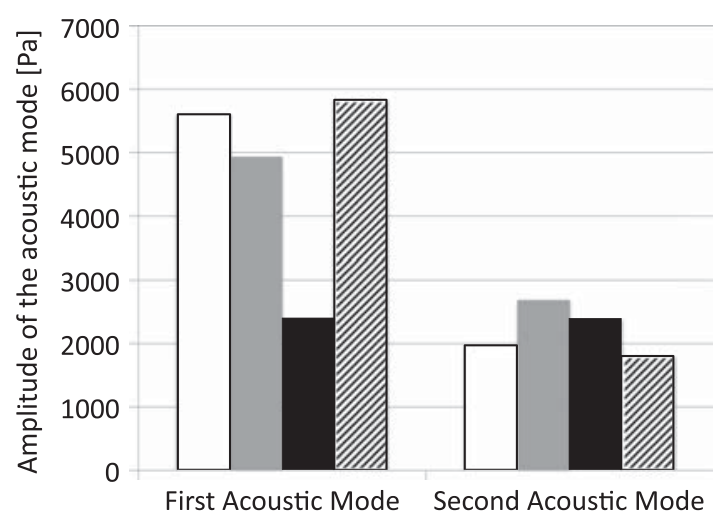

Figure 14. Amplitude of the acoustic mode for the case without FSI (white), without (grey) and with (black) modes crossing and dissipative materials such as $D=10^{-5} \mathrm{~K}$, with modes crossing and less-dissipative materials $D=10^{-7} \mathrm{~K}$ (hatched).

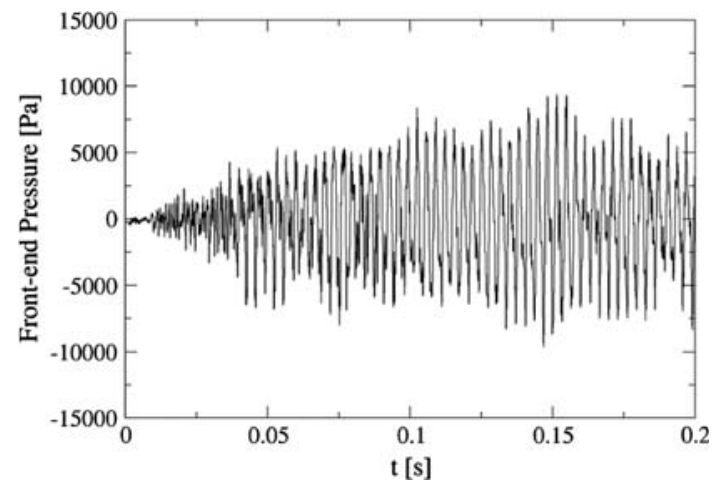

Figure 15. Pressure fluctuations at front-end for the case with modes crossing of the acoustic and structural modes and less-dissipative materials.

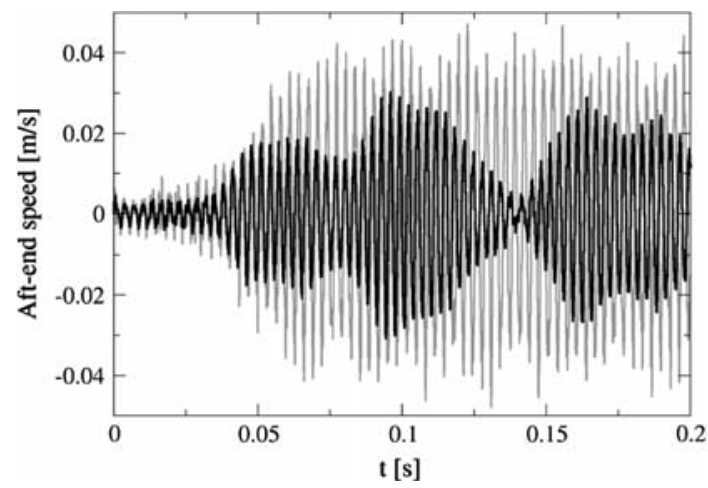

Figure 16. Velocity of the aft-end for the case of crossing between the acoustic and the structural modes for $D=10^{-7} \mathrm{~K}$ (grey line) and $D=10^{-5} \mathrm{~K}$ (black line). 
(cf. Figure 16), the amplitude of the acoustic modes are of the same order as in the reference case (cf. Figure 14). All other things being equal, these results highlight the impact of the dissipative nature of materials on the acoustics inside the combustion chamber.

\section{Conclusion}

A computational chain was developed that couples a fluid and a structure solver. Special care was taken to design the interface condition and the retained methodology showed good conservativity. The results obtained when considering an academic configuration for the coupling scheme were in full agreement with the theoretical developments. At last, the computational chain is applied to a subscaled version of the P230 SRM. Results on this configuration suggest that the structure may have an impact on the aero-acoustics instabilities, since it is able to dissipate acoustic energy when mode crossing between the structure and the acoustics occurs.

\section{Acknowledgements}

This work was granted access to the HPC resources of CINES under the allocation 2011-c2011026401 made by GENCI (Grand Equipement National de Calcul Intensif). The authors would like to thank Dr. Duchaine F., Jauré S. from CERFACS and Autrusson N., Bandelier E., Pevergne T. from Snecma Propulsion Solide for their help during the course of this study.

\section{References}

Batina, J.T. (1990). Unsteady Euler airfoil solutions using unstructured dynamic meshes. AIAA Journal, 28(8), 1381-1388.

Buis, S., Piacentini, A., \& Déclat, D. (2006). Palm: A computational framework for assembling highperformance computing applications. Concurrency and Computation: Practice and experience, 18 (2), 231-245.

Culick, F.E.C. (1966). Acoustic oscillations in solid propellant rocket chambers. Astronautica Acta, 12 (2), 113-126.

Dotson, K.W., \& Sako, B.H. (2004). An investigation of propulsion-structure interaction in solid rocket motors. 40th AIAA/ASME/SAE/ASEE Joint Propulsion Conference and Exhibit, 11-14 July 2004, Fort Lauderdale, Florida, AIAA Paper No. 2004-4183.

Fabignon, Y., Dupays, J., Avalon, G., Vuillot, F., Lupoglazoff, N., Casalis, G., \& Prevost, M. (2003). Instabilities and pressure oscillations in solid rocket motors. Aerospace Science and Technology, 7 (3), 191-200.

Farhat, C., Degand, C., Koobus, B., \& Lesoinne, M. (1998). Torsional springs for two-dimensional dynamic unstructured fluid meshes. Computer Methods in Applied Mechanics and Engineering, 163 (1-4), 231-245.

Farhat, C., \& Lesoinne, M. (1996). On the accuracy, stability, and performance of the solution of three dimensional nonlinear transient aeroelastic problems by partitioned procedures. Proceedings of the 37th AIAA/ASME/ASCE/AHS/ ASC Structures, Structural Dynamics and materials Conference, 1819 April 1996, Salt lake City, Utah, AIAA Paper No. 96-1388.

Flandro, G.A. (1986). Vortex driving mechanism in oscillatory rocket flows. Journal of Propulsion and Power, 2(3), 206-214.

Giordano, J., Jourdan, G., Burtschell, Y., Medale, M., Zeitoun, D.E., \& Houas, L. (2005). Shock wave impacts on deforming panel, an application of fluid-structure interaction. Shock Waves, 14(1), 103-110.

Hijlkema, J. Prévost, M., \& Casalis, G. (2011). On the importance of reduced scale Ariane 5 P230 solid rocket motor models in the comprehension and prevention of thrust oscillations. CEAS Space Journal, 1 (1), 99-107.

Hughes, T.J.R. (1987). The finite element method: Linear static and dynamic finite element analysis. Englewood Cliffs, NJ: Prentice-Hall.

Kourta, A. (1996a). Acoustic-mean flow interaction and vortex shedding in solid rocket motors. International Journal for Numerical Methods in Fluids, 22(6), 449-465.

Kourta, A. (1996b). Vortex shedding in segmented solid rocket motors. Journal of Propulsion and Power, 12(2), 371-376. 
Lesoinne, M., \& Farhat, C. (1993). Stability analysis of dynamic meshes for transient aeroelastic computations. 11th AIAA Computational Fluid Dynamics Conference, 6-9 July 1993, Orlando, Florida, AIAA Paper No. 93-3325.

Lesoinne, M., \& Farhat, C. (1996). Geometric conservation laws for flow problems with moving boundaries and deformable meshes, and their impact on aeroelastic computations. Computer Methods in Applied Mechanics and Engineering, 134(1-2), 71-90.

Lupoglazoff, N., \& Vuillot, F. (1996). Parietal vortex shedding as a cause of instability for long solid propellant motors numerical simulations and comparisons with firing tests. Office National d'Etudes et de Recherches Aerospatiales (ONERA), 92-Chatillon (France).

Nicoud, F., Benoit, L., Sensiau, C., \& Poinsot, T. (2007). Acoustic modes in combustors with complex impedances and multidimensional active flames. AIAA Journal, 45(2), 426.

Nielsen, E.J., \& Anderson, W.K. (2002). Recent improvements in aerodynamic design optimization on unstructured meshes. AIAA Journal, 40(6), 1155-1163.

Piperno, S., \& Farhat, C. (2001). Partitioned procedures for the transient solution of coupled aeroelastic problems-Part II: Energy transfer analysis and three-dimensional applications. Computer Methods in Applied Mechanics and Engineering, 190(24-25), 3147-3170.

Piperno, S., Farhat, C., \& Larrouturou, B. (1995). Partitioned procedures for the transient solution of coupled aroelastic problems Part I: Model problem, theory and two-dimensional application. Computer Methods in Applied Mechanics and Engineering, 124(1-2), 79-112.

Schmitt, P., Poinsot, T., Schuermans, B., \& Geigle, K.P. (2007). Large-eddy simulation and experimental study of heat transfer, nitric oxide emissions and combustion instability in a swirled turbulent high-pressure burner. Journal of Fluid Mechanics, 570, 17-46.

Schoenfeld, T., \& Rudgyardt, M. (1999). Steady and unsteady flow simulations using the hybrid flow solver AVBP. AIAA Journal, 37(11), 1378-1385.

Vuillot, F. (1995). Vortex-shedding phenomena in solid rocket motors. Journal of Propulsion and Power, $11(4), 626-639$.

Wasistho, B., Fiedler, R., Namazifard, A., \& Mclay, C. (2006). Numerical study of turbulent flow in SRM with protruding inhibitors. Urbana, 51, 61801. 